\title{
Electrochemical characterization of parylene-embedded carbon nanotube nanoelectrode arrays
}

\author{
Scott Miserendino ${ }^{1,3}$, Juhwan Yoo ${ }^{1}$, Alan Cassell ${ }^{2}$ and \\ Yu-Chong Tai ${ }^{1}$ \\ ${ }^{1}$ California Institute of Technology, 1200 East California Boulevard, Pasadena, CA 91125, \\ USA \\ ${ }^{2}$ NASA Ames Research Center, Moffett Field, CA 94035, USA \\ E-mail: scott@mems.caltech.edu
}

Received 20 July 2005, in final form 23 September 2005

Published 25 January 2006

Online at stacks.iop.org/Nano/17/S23

\begin{abstract}
A novel parylene-embedded carbon nanotube nanoelectrode array is presented for use as an electrochemical detector working electrode material. The fabrication process is compatible with standard microfluidic and other MEMS processing without requiring chemical mechanical polishing. Electrochemical studies of the nanoelectrodes showed that they perform comparably to platinum. Electrochemical pretreatment for short periods of time was found to further improve performance as measured by cathodic and anodic peak separation of $\mathrm{K}_{3} \mathrm{Fe}(\mathrm{CN})_{6}$. A lower detection limit below $0.1 \mu \mathrm{M}$ was measured and with further fabrication improvements detection limits between $100 \mathrm{pM}$ and $10 \mathrm{nM}$ are possible. This makes the nanoelectrode arrays particularly suitable for trace electrochemical analysis.
\end{abstract}

(Some figures in this article are in colour only in the electronic version)

\section{Introduction}

Electrochemical detectors (ECDs) are a key component in high performance liquid chromatography (HPLC) systems. Onchip HPLC systems ( $\mu$ HPLC) use relatively small sample volumes compared to conventional HPLC while offering comparable separation performance [1]. The small sample volumes, however, have insufficient analytes for simple UV absorption detection. ECDs offer a viable alternative for electrochemically active analytes with the advantage of enhanced selectivity in detection. This detection method is ideal for use in $\mu$ HPLC systems since it responds to analyte concentration without regard for the total amount of analyte. ECDs with detection limits as low as $0.1 \mathrm{pM}$ have been reported under special conditions but practical limits over a wide variety of analytes tend to be above $0.1 \mu \mathrm{M}[2,3]$. Also, it has been reported that nanoarrays of vertical carbon nanotubes can achieve detection limits on the order of $1 \mathrm{nM}$ [4]. The development of ultra-low detection limit ECDs coupled with

\footnotetext{
3 Author to whom any correspondence should be addressed.
}

an $\mu \mathrm{HPLC}$ will facilitate selective measurement of analytes produced by a single living cell.

Integration of carbon nanotubes (CNTs) with microfluidic HPLC columns presents several major fabrication challenges. As grown, vertical carbon nanotubes cannot withstand standard MEMS processing such as photolithography. The vertical carbon nanotubes must be encased in a stabilizing matrix prior to the fabrication of the microfluidic channels that will deliver the analyte. In the past, thermal chemical vapour deposition (CVD) of tetraethylorthosilicate (TEOS) has been used as a stabilizing matrix, which requires the use of chemical mechanical polishing (CMP) to planarize the electrode surface and expose the tips of the carbon nanotubes [4-8]. The high temperature CVD TEOS process and accompanying CMP can make this technique expensive, time consuming, and incompatible with other processing requirements for on-chip fluidic channels. To address these limitations, a process based on room temperature CVD deposition of poly(monochloro$p$-xylylene), known by its commercial name Parylene-C, has been developed to replace CVD TEOS. Parylene can conformally coat the CNTs and be etched back using $\mathrm{O}_{2}$ 
(a)

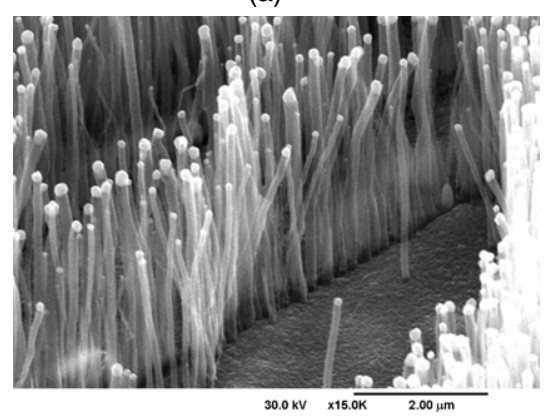

(b)

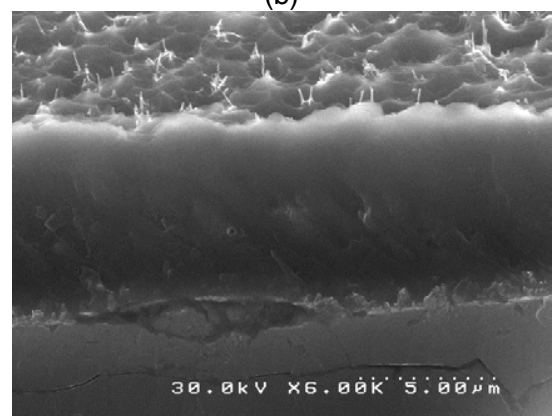

Figure 1. (a) CNTs immediately after growth. (b) Cross-section of CNT electrode array. The cross-section image shows the silicon wafer with approximately $7 \mu \mathrm{m}$ of parylene embedded CNTs. Approximately $0.5 \mu \mathrm{m}$ of the CNTs are exposed above the surface of the parylene. SEM images were taken at a $59^{\circ}$ tilt angle relative to the surface of the parylene.

(a)

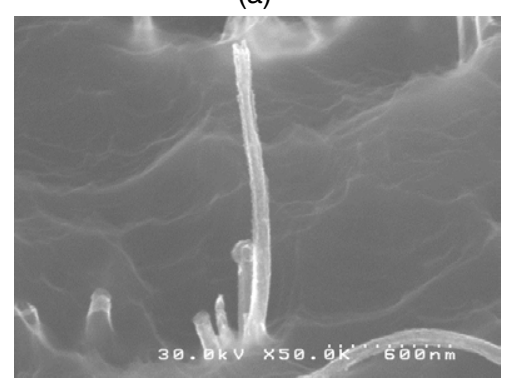

(b)

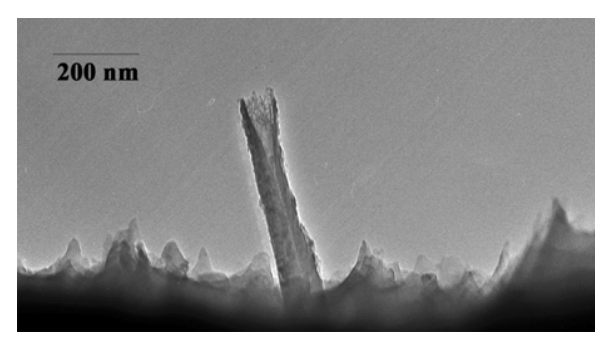

Figure 2. (a) SEM of a single CNT tip. (b) TEM of a single CNT tip. Note the removal of an Ni seed at the top of the CNT and the removal of parylene from the exposed tip. The SEM image was taken at a $59^{\circ}$ tilt angle.

plasma to expose the CNT tips. This parylene process completely removes the need for CVD TEOS and CMP.

\section{Fabrication}

\subsection{CNT nanoarray electrodes}

CNT nanoarrays are grown on $100 \mathrm{~mm}$ diameter silicon wafers. The steps involved in full-wafer electrode fabrication are the following.

(1) Metal film deposition. A $50 \mathrm{~nm}$ barrier layer of Ti was deposited using electron beam evaporation on a $\mathrm{Si}$ wafer.

(2) Catalyst deposition. A $35 \mathrm{~nm}$ layer of $\mathrm{Ni}$ was deposited using electron beam evaporation for use as the CNT growth catalyst.

(3) PECVD CNT growth. Vertically aligned multi-walled carbon nanotubes (MWCNTs) were grown for $30 \mathrm{~min}$ from the $\mathrm{Ni}$ catalyst in a variety of processing conditions. The processing gas used was a mixture of $\mathrm{NH}_{3}$ and $\mathrm{C}_{2} \mathrm{H}_{2}$. The highest quality CNT growth was achieved with a $\mathrm{NH}_{3}$ to $\mathrm{C}_{2} \mathrm{H}_{2}$ ratio of $110: 25$ mTorr and $1510 \mathrm{~W}$ of power. Average CNT height and diameter were determined using scanning electron microscopy (SEM) to be approximately $5-8 \mu \mathrm{m}$ and $100 \mathrm{~nm}$, respectfully. A complete characterization of the growth process has been previously reported [4-8].

(4) Parylene deposition and reflow. Approximately $7 \mu \mathrm{m}$ of parylene-C (Specialty Coating Systems, Indianapolis, IN) was deposited on the wafer. The parylene deposition was carried out at room temperature using a PDS 2010 Labcoter (Specialty Coating Systems, Indianapolis, IN). After deposition, the wafer was heated to temperatures between 350 and $375^{\circ} \mathrm{C}$ for one hour in a $\mathrm{N}_{2}$ atmosphere to allow the parylene to reflow and planarize the surface of the electrode. The wafer was then allowed to cool at a rate of $1^{\circ} \mathrm{C} \mathrm{min}-1$. Figure 1(a) shows CNTs before parylene deposition.

(5) CNT tip exposure. The parylene surface was exposed to $\mathrm{O}_{2}$ plasma to etch several microns of parylene and reveal CNT tips forming the nanoelectrode array. Figure 1(b) shows a cross-section of the CNT array after completion of the fabrication process. Variations in CNT height in the growth step allowed average tip to tip separation to be controlled through manipulating the $\mathrm{O}_{2}$ plasma conditions and etch time. The exposed CNT length was measured to be up to $1 \mu \mathrm{m}$ using SEM and transmission electron microscopy (TEM) (figure 2). SEM and TEM images show that the $\mathrm{O}_{2}$ plasma can leave some parylene residue on the exposed stalk. Longer etching times reduce the amount of residue.

(6) Acid etch. The CNT tips were etched in 1:1:2 $\left(\mathrm{HNO}_{3}: \mathrm{H}_{2} \mathrm{SO}_{4}: \mathrm{H}_{2} \mathrm{O}\right)$ for $5 \mathrm{~min}$ to remove the Ni seeds.

(7) Dicing. The wafer was diced into individual electrode arrays approximately $10 \times 20 \mathrm{~mm}^{2}$.

Figure 3 shows a cross-sectional diagram of the process after steps (2), (3), (4), and (6). A variety of $\mathrm{O}_{2}$ etch times were tested to achieve the desired tip to tip separation distance of approximately $1 \mu \mathrm{m}$ measured using SEM (figure 4). 


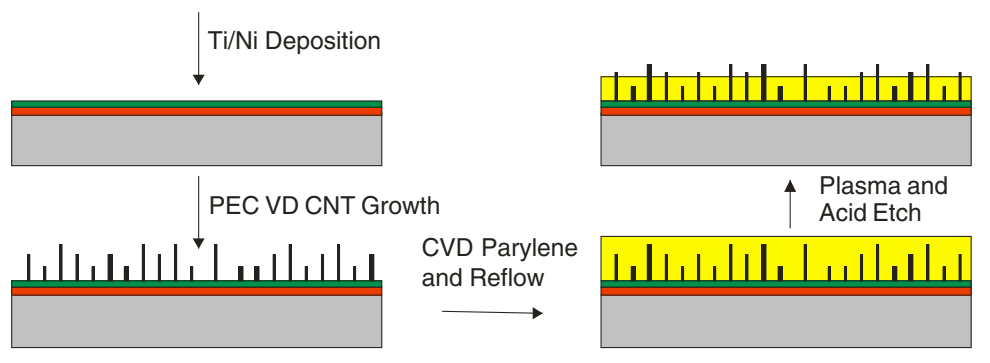

Figure 3. Fabrication process flow for CNT nanoarray electrodes.

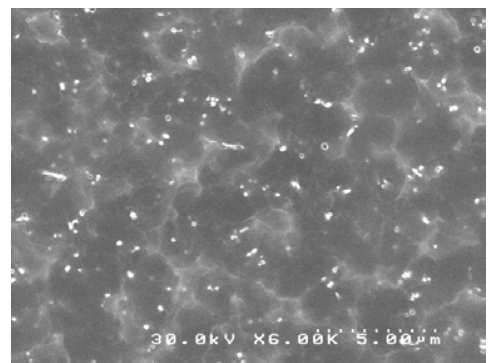

Figure 4. Overhead view of CNT electrode array. CNT tip to tip separation is estimated to be approximately $1 \mu \mathrm{m}$.

\subsection{CNT electrode film patterning}

Two methods were employed to pattern the CNT electrode film. The first method used was to pattern the Ti/Ni seed layer using lift-off lithography. CNTs were selectively grown in areas with the seed layer. The second method explored was to fabricate a complete CNT electrode film over the entire wafer and pattern that film using $\mathrm{O}_{2}$ plasma and a photoresist mask. The embedded CNT film etch rate was found to be approximately $0.1 \mu \mathrm{m} \mathrm{min}^{-1}$ when etched using a reactive ion etching (RIE) system, three times slower than that of regular parylene. Both methods successfully produced patterned CNT films.

\section{Results}

\subsection{Electrochemical characterization}

All electrochemical measurements were made using a standard three-electrode configuration. The parylene-embedded CNT nanoarrays were used as a working electrode. Platinum wire was the auxiliary electrode. $\mathrm{An} \mathrm{Ag} / \mathrm{AgCl}, \mathrm{KCl}$ (saturated) electrode served as the reference electrode in all experiments. A Princeton Applied Research model 263A potentiostat (Princeton, NJ) interfaced with PowerSuite software was used to conduct the cyclic voltammetry $(\mathrm{CV})$ and record data. $\mathrm{K}_{3} \mathrm{Fe}(\mathrm{CN})_{6}$ was used as received from SigmaAldrich (St Louis, MO). Stock solution of $100 \mathrm{mM} \mathrm{K}_{3} \mathrm{Fe}(\mathrm{CN})_{6}$ in $0.1 \mathrm{M} \mathrm{KCl}$ was diluted with additional $0.1 \mathrm{M} \mathrm{KCl}$ to obtain the desired concentrations of $\mathrm{K}_{3} \mathrm{Fe}(\mathrm{CN})_{6}$.

The CNT nanoarrays were electrochemically characterized by cyclic voltammetry in $5.0 \mathrm{mM} \mathrm{K}_{3} \mathrm{Fe}(\mathrm{CN})_{6}$ with $0.1 \mathrm{M}$ $\mathrm{KCl}$ at a scan rate of $100 \mathrm{mV} \mathrm{s}^{-1}$. The anodic and cathodic peak separation $\left(\Delta E_{\mathrm{p}}\right)$ was used as the figure of merit for comparison of electrode performance. For a Nernstian reaction,

$$
\Delta E_{\mathrm{p}}=\frac{2.3 R T}{n F},
$$

where $R$ is the ideal gas law constant, $T$ is temperature, $n$ is the number of electrons transferred in the reduction-oxidation (redox) reaction, and $F$ is Faraday's constant [3]. At room temperature for a single-electron redox reaction this expression evaluates to roughly $59 \mathrm{mV}$. Figure 5 shows typical CV scans for platinum, non-isolated CNT nanoarrays, and isolated CNT nanoarrays. Isolated CNT electrodes were attached to an acrylic backing and had all exposed edges covered with epoxy. The isolation protocol improved peak separation from $450 \mathrm{mV}$ in the non-isolated sample to $196 \mathrm{mV}$ in the isolated sample. The isolated CNT electrodes performed comparably to platinum.

\subsection{Pretreatment}

Various electrochemical pretreatment protocols have been shown to improve electron transfer kinetics on glassy carbon electrodes $[9,10]$. The similarity in structure between glassy and graphitic carbon and MWCNTs suggested that similar pretreatment protocols may also be beneficial [5]. To further improve the performance of the isolated CNT nanoelectrodes, a variety of electrochemical pretreatments were tested. Before any isolated nanoelectrode was pretreated a $\mathrm{CV}$ was taken in $5.0 \mathrm{mM} \mathrm{K} \mathrm{K}_{3} \mathrm{Fe}(\mathrm{CN})_{6}$ with $0.1 \mathrm{M} \mathrm{KCl}$. Only electrodes with an initial $\Delta E_{\mathrm{p}}=200 \pm 20 \mathrm{mV}$ were used to test pretreatment conditions. Each treatment was performed on an isolated CNT nanoelectrode before a CV scan was conducted to determine the pretreated $\Delta E_{\mathrm{p}}$ value (table 1). The results show pretreatments of $\geqslant 30 \mathrm{~s}$ lead to degraded electrode performance compared to untreated electrodes. It is possible that electrochemical pretreatments $\geqslant 30 \mathrm{~s}$ etch most of the exposed CNT tips causing the observed degradation in performance. CNT tip heights were observed under SEM before and after pretreatments of $30 \mathrm{~s}$ and were found to be substantially shorter after the pretreatments. In most cases, tips were levelled to the parylene surface after $30 \mathrm{~s}$ of pretreatment. Fifteen second pretreatments in $\mathrm{H}_{2} \mathrm{SO}_{4}, \mathrm{HCl}$, and $\mathrm{HNO}_{3}$ improved electrode performance. The improvement in performance is possibly due to reduction of oxides and remaining parylene residue on the surface of the carbon. A $0.8 \mathrm{~V}$ pretreatment for $15 \mathrm{~s}$ in $\mathrm{HNO}_{3}$ gave the best peak separation. 


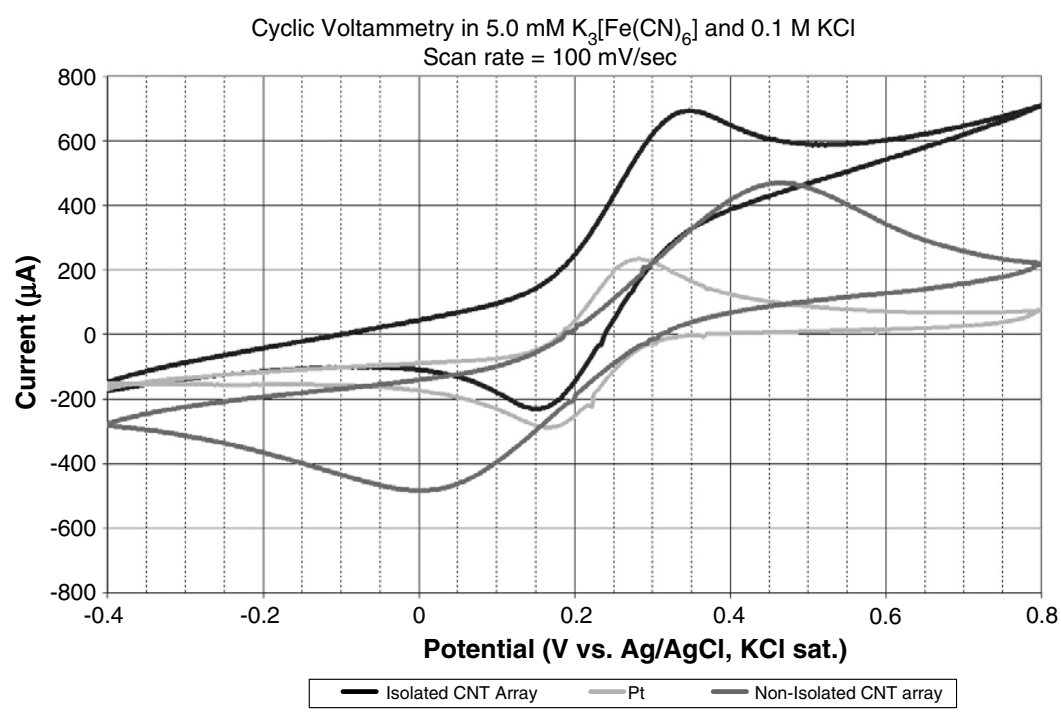

Figure 5. Cyclic voltammetry in $5.0 \mathrm{mM} \mathrm{K}_{3} \mathrm{Fe}(\mathrm{CN})_{6}$ and $0.1 \mathrm{M} \mathrm{KCl}$ at a scan rate of $100 \mathrm{mV} \mathrm{s}^{-1}$ for three different electrode materials. Pt is included as a standard and has a $\Delta E_{\mathrm{p}}=120 \mathrm{mV}$. The non-isolated CNT array has a $\Delta E_{\mathrm{p}}=450 \mathrm{mV}$. The isolated CNT array has a $\Delta E_{\mathrm{p}}=196 \mathrm{mV}$.
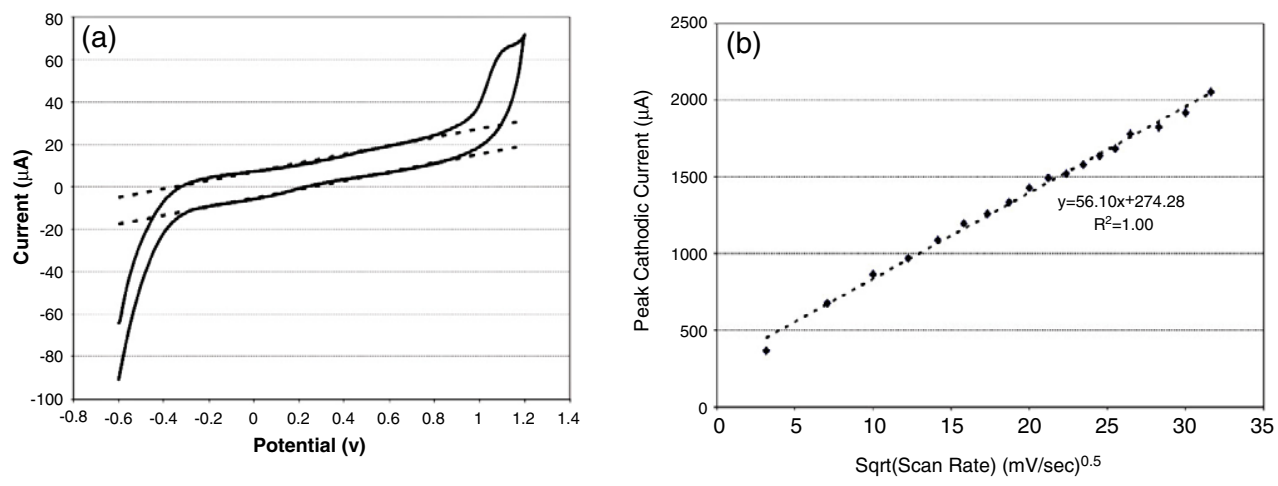

Figure 6. (a) $\mathrm{CV}$ scan of $0.1 \mathrm{M} \mathrm{KCl}$ at a scan rate of $100 \mathrm{mV} \mathrm{s}^{-1}$. No significant water hydrolysis occurs between -0.35 and $1.0 \mathrm{~V}$. The dashed lines show the cathodic and anodic baseline currents determined by a linear fit in the potential window. (b) Peak cathodic current versus the square root of scan rate for scan rates between $10 \mathrm{mV} \mathrm{s}^{-1}$ and $1 \mathrm{~V} \mathrm{~s}^{-1}$. The dashed line is a linear fit of the data points showing a nearly perfect linear dependence.

Table 1. $\Delta E_{\mathrm{p}}$ of $\mathrm{K}_{3} \mathrm{Fe}(\mathrm{CN})_{6}$ after electrochemical pretreatment of CNT nanoelectrodes.

\begin{tabular}{llll}
\hline $1.0 \mathrm{M} \mathrm{HNO}_{3}$ & $0.6 \mathrm{~V} / 30 \mathrm{~s}$ & $0.8 \mathrm{~V} / 15 \mathrm{~s}$ & $\begin{array}{l}1 \mathrm{~V} / 30 \mathrm{~s} \\
\mathrm{NR}^{\mathrm{a}}\end{array}$ \\
\hline$\Delta E_{\mathrm{p}}(\mathrm{mV})$ & 469 & 123 & ${ }^{\mathrm{M}}$ \\
\hline $1.0 \mathrm{MCl}$ & $0.6 \mathrm{~V} / 30 \mathrm{~s}$ & $0.8 \mathrm{~V} / 15 \mathrm{~s}$ & $1 \mathrm{~V} / 30 \mathrm{~s}$ \\
$\Delta E_{\mathrm{p}}(\mathrm{mV})$ & $\mathrm{NT}^{\mathrm{b}}$ & 174 & 483 \\
\hline $1.0 \mathrm{M} \mathrm{H}_{2} \mathrm{SO}_{4}$ & $0.6 \mathrm{~V} / 30 \mathrm{~s}$ & $0.8 \mathrm{~V} / 15 \mathrm{~s}$ & $1 \mathrm{~V} / 30 \mathrm{~s}$ \\
$\Delta E_{\mathrm{p}}(\mathrm{mV})$ & 922 & 167 & 870 \\
\hline $1.0 \mathrm{M} \mathrm{KOH}$ & $0.6 \mathrm{~V} / 30 \mathrm{~s}$ & $0.8 \mathrm{~V} / 15 \mathrm{~s}$ & $1 \mathrm{~V} / 30 \mathrm{~s}$ \\
$\Delta E_{\mathrm{p}}(\mathrm{mV})$ & $\mathrm{NT}^{\mathrm{b}}$ & 224 & 856 \\
\hline
\end{tabular}

a Tested but no response.

${ }^{\mathrm{b}}$ Not tested.

\subsection{Additional electrochemical characterizations after pretreatment}

Two electrodes were prepared using optimal processing parameters, isolated, and electrochemically pretreated in $1.0 \mathrm{M}$
$\mathrm{HNO}_{3}$ at $0.8 \mathrm{~V}$ for $15 \mathrm{~s}$. Solutions of $5.0 \mathrm{mM} \mathrm{K}{ }_{3} \mathrm{Fe}(\mathrm{CN})_{6}$ in a supporting electrolyte of $0.1 \mathrm{M} \mathrm{KCl}$, phosphate buffered

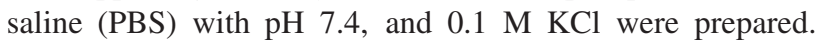
The potential window of the electrode whose boundaries were defined by the onset potentials of water hydrolysis was measured in both $0.1 \mathrm{M} \mathrm{KCl}$ and PBS. The water potential window in the $0.1 \mathrm{M} \mathrm{KCl}$ was determined to be between approximately -0.35 and $1.0 \mathrm{~V}$ (figure 6(a)). The potential window in PBS was determined to be approximately -0.6 to $1.1 \mathrm{~V}$. The potential window of the electrode in $0.1 \mathrm{M} \mathrm{KCl}$ and PBS was determined to be similar to that of $\mathrm{Pt}$ in $1 \mathrm{M} \mathrm{H}_{2} \mathrm{SO}_{4}$ and $\mathrm{pH} 7$ buffer, respectfully [3]. The $0.1 \mathrm{M} \mathrm{KCl}$ window is slightly smaller than that reported for glassy carbon, -1 to $1 \mathrm{~V}$, in $0.1 \mathrm{M} \mathrm{KCl}[2,3]$. The double-layer capacitance, $C_{\mathrm{dl}}$, of the electrode can be estimated from the baseline currents and was found to be approximately 51 and $47 \mu \mathrm{F} \mathrm{cm}^{-2}$ in $0.1 \mathrm{M} \mathrm{KCl}$ and PBS, respectively. The measured $C_{\mathrm{dl}}$ is consistent with the expected values for a glassy carbon electrode or the edge plane of a highly ordered pyrolytic graphite electrode [2]. All 
Electrochemical characterization of parylene-embedded carbon nanotube nanoelectrode arrays

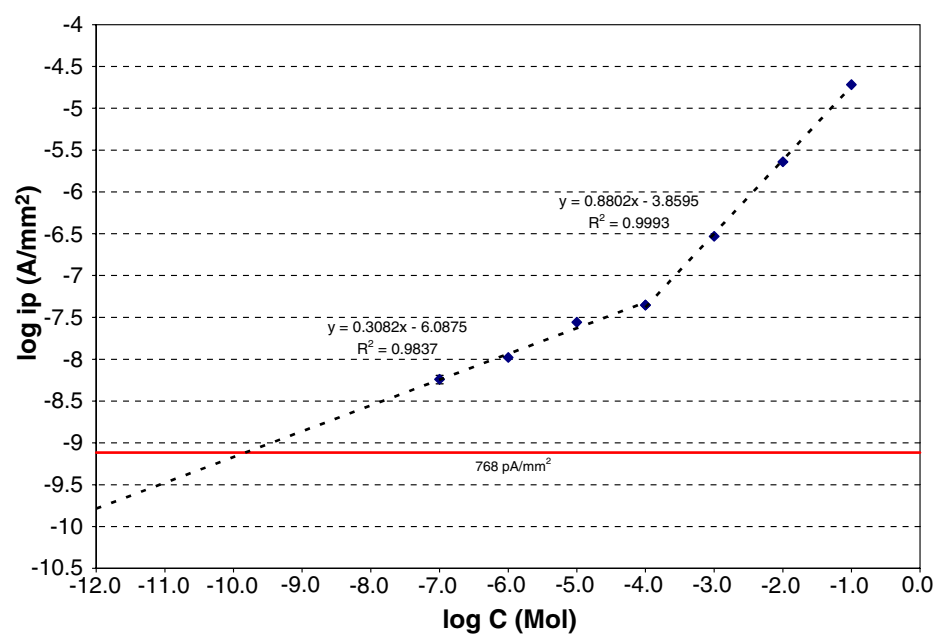

Figure 7. Plot of the $\log _{10}$ of the measured peak cathodic current relative to background current verses $\log _{10}$ of the concentration of $\mathrm{K}_{3} \mathrm{Fe}(\mathrm{CN})_{6}$. The low concentration linear fit (dashed line) has been extended to show its intersection with the lower detection limit (solid red line). This indicates that the lower detection limit could possibly be extended to be on the order of $100 \mathrm{pM}$. Using current fabrication techniques no signal was detectable for concentrations below $100 \mathrm{nM}$.

normalized values were calculated using the geometric surface area of the electrode submerged in solution.

Using the second electrode, $\mathrm{CV}$ scans of the $5.0 \mathrm{mM}$ $\mathrm{K}_{3} \mathrm{Fe}(\mathrm{CN})_{6}$ solution were taken at scan rates ranging from $10 \mathrm{mV} \mathrm{s}^{-1}$ to $1 \mathrm{~V} \mathrm{~s}^{-1}$. For each CV scan, the baseline current due to the uncompensated resistance was determined by a linear fit of the voltammagram in the -0.1 to $0.1 \mathrm{~V}$ range where no electrochemical activity was observed. The cathodic peak current was measured from the baseline to the voltammagram. For a Nernstian reaction at $25^{\circ} \mathrm{C}$ the expected peak current, $i_{\mathrm{p}}$, is

$$
i_{\mathrm{p}}=\left(2.69 \times 10^{5}\right) n^{3 / 2} A D^{1 / 2} C v^{1 / 2}
$$

where $n$ is the number of electrons transferred in the redox reaction, $A$ is the surface area of the electrode, $D$ is the diffusivity of the analyte, $C$ is the bulk analyte concentration, and $v$ is the scan rate [3]. Figure 6(b) shows that the electrode performed as expected, exhibiting a near perfect linear relationship between the peak cathodic current and the square root of the scan rate.

\subsection{Sensitivity and lower detection limit}

Solutions of concentrations of $\mathrm{K}_{3} \mathrm{Fe}(\mathrm{CN})_{6}$ ranging from $0.1 \mu \mathrm{M}$ to $100 \mathrm{mM}$ in a supporting electrolyte of $0.1 \mathrm{M} \mathrm{KCl}$ were prepared. For each concentration a CV scan was taken at a scan rate of $100 \mathrm{mV} \mathrm{s}^{-1}$ and the baseline current due to the uncompensated resistance was determined by a linear fit of the voltammagram in the -0.1 to $0.1 \mathrm{~V}$ range where no electrochemical activity was observed. The cathodic peak current was measured from the baseline to the voltammagram. According to equation (2), a plot of the $\log _{10} i_{\mathrm{p}}$ verse $\log _{10} C$ will produce a line with unit slope. Figure 7 shows a graph of $\log _{10} i_{\mathrm{p}}$ versus $\log _{10} C$ obtained using a CNT electrode. One linear fit is made for concentrations above $100 \mu \mathrm{M}$ and another for concentrations below $100 \mu \mathrm{M}$. The two fits cross at approximately $100 \mu \mathrm{M}$. The high concentration fit has a slope of 0.88 and the low concentration fit has a slope of 0.308 . Both of the slopes are significantly lower than the expected value of unity. At higher concentrations the sensitivity is much closer to the expected linear dependence than at lower concentrations. These results, however, are consistent with previously reported behaviour of similar vertical CNT electrodes and other nanoelectrode arrays $[4,11]$.

A lower detection limit was determined based on the noise in the baseline current fit. We define a detectable signal to be any current level above twice the root mean square error (RMSE) in the linear baseline fit. The average RMSE was used to calculate the overall lower detection limit of the electrode which was found to be approximately $768 \mathrm{pA} \mathrm{mm}^{-2}$. Assuming the experimentally determined relationship between $i_{\mathrm{p}}$ and $C$ will hold to concentrations below $0.1 \mu \mathrm{M}$ we estimate that a lower detection limit on the order $100 \mathrm{pM}$ is possible.

\section{Conclusion}

Parylene-embedded carbon nanotube nanoelectrode arrays have the potential for ultra-low detection limits while being relatively easy to produce using standard MEMS processes after the CNT growth. The CNT electrode films can be patterned either before growth or after being embedded in parylene. The nanoelectrodes have demonstrated stable electrochemical behaviour. The peak separation was found to be comparable to Pt but the CNT electrodes exhibit a higher background current due to their greater resistivity. A variety of electrochemical pretreatments were tested and several were found to improve electrode performance.

Electrode sensitivity was measured and found to have a nonlinear dependence on bulk concentration. Koehne also observed a similar nonlinear relationship where $i_{\mathrm{p}}$ was proportional to $C^{\alpha}$ with reported exponents, $\alpha$, between 0 and 0.2. Koehne theorized that the stabilizing matrix could be adsorbing analyte on its surface, leading to local concentrations near the CNTs higher than that in the bulk solution $[4,11]$. The $C^{\alpha}$ relationship results in lower sensitivity which is normally undesirable. One notable benefit, however, to the $C^{\alpha}$ relationship is that it favours lower detection limits, since a 
larger decrease in analyte concentration is necessary before the detected signal is reduced below the background noise level. This nonlinear relationship reduced the electrode sensitivity but improved its lower detection limit. Although the tested electrode did not produce a detectable signal when used in $10 \mathrm{nM}$ solution, it is probable that through further refinement of the fabrication process an improvement in lower detection limit of one to three orders of magnitude can be achieved. Due to their lower than average detection limit the CNT nanoelectrode films are well suited for use in trace electrochemical analysis. Because of the compatibility with other MEMS fabrication techniques the CNT nanoelectrodes will be integrated as an electrochemical detector in integrated $\mu$ HPLC systems.

\section{Acknowledgment}

This work was supported by the Center for Cell Mimetic Space Exploration (CMISE), a NASA University Research, Engineering and Technology Institute (URETI), under award number NCC 2-1364.

\section{References}

[1] He Q, Pang C, Tai Y C and Lee T 2004 MEMS'04 Technical Digest pp 212-5

[2] Kissinger P and Heineman W (ed) 1996 Laboratory Techniques in Electroanalytical Chemistry 2nd edn (New York: Dekker)

[3] Bard A and Faulkner L 2001 Electrochemical Methods: Fundamentals and Applications 2nd edn (New York: Wiley)

[4] Koehne J, Li J, Cassell A, Chen H, Ye Q, Ng H T, Han J and Meyyappan M 2004 J. Mater. Chem. 14 676-84

[5] Li J, Cassell A, Delzeit L, Han J and Meyyappan M 2002 J. Phys. Chem. B 106 9299-305

[6] Li J, Stevens R, Delzeit L, Ng H T, Cassell A, Han J and Meyyappan M 2002 Appl. Phys. Lett. 81 910-2

[7] Li J, Ng H T, Cassell A, Fan W, Chen H, Ye Q, Koehne J and Meyyappan M 2003 Nano Lett. 3 597-602

[8] Koehne J, Chen H, Li J, Cassell A, Ye Q, Ng H T, Han J and Meyyappan M 2003 Nanotechnology 14 1239-45

[9] Engstrom R 1982 Anal. Chem. 54 2310-4

[10] Bellby A, Sasaki T and Stern H 1995 Anal. Chem. 67 976-80

[11] Menon V and Martin C 1995 Anal. Chem. 67 1920-8 